\title{
Kommunikationskonzepte und Kommunikationsverben
}

\section{Edeltraud Winkler}

\section{Einführung}

Die folgenden Ausführungen orientieren sich hauptsächlich an der Praxis der Beschreibung eines bestimmten Lexikonausschnittes des Deutschen. Daher stehen auch vornehmlich Kommunikationsverben und deren Besonderheiten zur Debatte; um Kommunikationskonzepte beziehungsweise Modelle sprachlicher Kommunikation kann es nur insofern gehen, als sie Grundlage und Hintergrund für eine Beschreibung der entsprechenden Verben sind. Die Beispiele sowie die gewählte Beschreibungssprache beziehen sich auf ein Projekt, das die synonymischen Beziehungen kommunikativer Ausdrücke des Deutschen beschreibt und erklärt, darüber hinaus aber noch eine ganze Reihe anderer wichtiger Gesichtspunkte von Kommunikationsverben und ihrer Strukturierung beinhaltet. Weitere Einzelheiten über das Projekt ESKA (Erklärende Synonymik kommunikativer Ausdrücke des Deutschen) finden sich in Harras (1991), Harras und Winkler (1994) sowie Winkler (1995).

Der Beschreibungsrahmen für Kommunikationsverben kann nun unterschiedlich sein. Sie können sowohl in ihrer Gesamtheit als auch einzeln beziehungsweise unter bestimmten Gesichtspunkten in Gruppen zusammengefaßt beschrieben werden. Allein daraus ergeben sich verschiedene Ebenen der Beschreibung, die nicht nur in hierarchischer Beziehung zueinander stehen. Vier solcher Ebenen, nämlich der allgemeine Rekurssituationstyp, spezielle Rekurssituationstypen, Wortfelder und Lexikoneinträge, sollen im folgenden genauer beschrieben und ihre Beziehungen zueinander dargestellt werden. Um einen einheitlichen Bezugspunkt zu haben und eine bessere Vorstellung vom Zusammenwirken der verschiedenen Beschreibungsebenen vermitteln zu können, sind die angeführten Beispiele fast ausschließlich dem Bereich der Fragen und der Aufforderungen entnommen worden.

\section{Ausgangspunkt der Beschreibung: die Grundfunktion von Kommunikationsverben}

Machen wir uns zunächst einmal mit der Funktion der untersuchten sprachlichen Einheiten vertraut. Kommunikationsverben sind Verben, die der Bezug- 
nahme auf kommunikative Handlungen, also der Bezugnahme auf sprachliche Äußerungsakte dienen. Es handelt sich um sprachliche Einheiten, die eine doppelte Funktion ausüben: Sie werden, wie alle anderen sprachlichen Einheiten auch, in der sprachlichen Kommunikation zur Übermittlung und als Träger bestimmter Informationen verwendet, und sie dienen gleichzeitig der Beschreibung und der Bezugnahme auf sprachliche Kommunikation, was andere sprachliche Einheiten nicht oder nicht in dem Maße können. Bezugnahme auf sprachliche Kommunikation heißt hier, daß ein Sprecher innerhalb eines Kommunikationsereignisses auf ein anderes (vergangenes, zukünftiges oder eventuell auch gleichzeitig stattfindendes) Kommunikationsereignis Bezug nimmt und dieses aus seiner Sicht einordnet und bewertet, indem er es mit einem bestimmten Kommunikationsverb benennt. Dabei nimmt er natürlich nicht nur isoliert auf eine einzelne Äußerung Bezug, sondern auch auf die Gesamtheit der situativen Bedingungen, unter denen die Äußerung stattfand. Unter Umständen kann der Sprecher sich auch auf seine eigenen oder jedenfalls auf Äußerungen beziehen, die in der gegenwärtigen Kommunikationssituation stattfinden. Es handelt sich dann um eine sogenannte äußerungskommentierende, gesprächssteuernde oder gesprächsgliedernde, manchmal auch explizit wertende Funktion, die diese metakommunikativen Äußerungen und die in ihnen enthaltenen Kommunikationsverben dadurch verliehen bekommen. Durch den Gebrauch solcher äußerungskommentierender Ausdrücke will der Sprecher sicherstellen, daß er so, wie es von ihm intendiert war, verstanden wird, und auf diese Weise Mißverständnisse und Konflikte vermeiden beziehungsweise bereits bestehende Kommunikationskonflikte entschärfen oder beseitigen. (Daneben gibt es natürlich auch metakommunikative Äußerungen, die keine Kommunikationsverben enthalten; diese spielen für die hier vorgestellten Betrachtungen keine Rolle.) Beispiele für solche selbstbezüglichen Verwendungen wären:

(1) Ich will zunächst das Problem darstellen und danach die Frage beantworten, welche Lösungswege die geeignetsten sind.

(2) Fassen wir noch einmal zusammen: ...

(3) Unter uns gesagt, ich halte das Ganze für einen gut eingefädelten Trick von Peter, um sich interessant zu machen.

(4) Ich habe Ihnen das nicht vorgeworfen, ich habe diesen Punkt nur zum besseren Verständnis erwähnt.

(5) Ich behaupte das jetzt einfach mal.

Solche und ähnliche Äußerungen dienen der Gliederung des Kommunikationsablaufs, sie leiten einen neuen Abschnitt ein, ordnen den Gesprächsverlauf, haben resümierende Funktion oder legen den Status bestimmter Äußerungen fest, wozu mitunter auch eine explizite Wertung von einzelnen Äußerungen gehören kann. Bei diesen Anmerkungen zu dem Sonderfall der Bezugnahme mit Kommunikationsverben will ich es belassen. 
Im Normalfall (oder jedenfalls im häufigeren Fall) besteht die Grundfunktion von Kommunikationsverben in der Bezugnahme mit sprachlichen Mitteln auf außerhalb des gegenwärtigen Kommunikationsereignisses stattfindende Äußerungen. Dazu gehören Beispiele wie die folgenden:

(6) Er hat sie gebeten, pünktlich zu sein.

(7) Felix hat mir mitgeteilt, daß er heute leider nicht kommen kann.

(8) Die Zeitungen berichten ausführlich über die Haushaltsdebatte im Bundestag.

(9) Fritz hat sich bei seinem Professor darüber beklagt, daß zu wenig Seminare zu diesem Themenkreis angeboten würden.

(10) Paul hat versprochen, das Buch morgen zurückzubringen.

Mit Sätzen wie den eben genannten wird mehr oder weniger explizit auf andere Äußerungen Bezug genommen. In manchen Fällen bleibt die Bezugnahme mehr auf die Äußerung beschränkt, in anderen wird die Gesamtsituation viel stärker einbezogen. Aber auch wenn hauptsächlich auf die Äußerung Bezug genommen wird, kommt die Situation durch die Verbwahl mit ins Spiel, denn die Wahl des Verbs muß zwangsläufig wichtige Merkmale der jeweiligen Situation berücksichtigen.

\section{Die Bedeutungsinvariante für alle Kommunikationsverben}

Aus der angegebenen Grundfunktion, der Bezugnahme auf sprachliche Äußerungen, läßt sich für die Kommunikationsverben als Verbklasse eine Bedeutungsinvariante, ein einheitlicher Bedeutungskern ableiten. Dazu gehört in jedem Falle die Tatsache, daß es einen Sprecher $S$ gibt (der aber keine Einzelperson zu sein braucht, sondern auch in Form einer Institution oder ähnlichem auftreten kann), der einem Hörer $H$ gegenüber eine sprachliche Äußerung $S a$ produziert. Diese Äußerung besitzt im Regelfall einen propositionalen Gehalt $P$. (Lediglich bei einigen wenigen Äußerungen wie "Guten Tag!" und ähnlichen kann man begründet davon ausgehen, daß sie keinen propositionalen Gehalt haben.) Mit der Äußerung verbindet der Sprecher zumeist eine bestimmte Einstellung $E(S)$, die er mit Hilfe der Äußerung transportieren will. Es kann hier selbstverständlich immer nur um ausgedrückte Sprechereinstellungen gehen; Einstellungen, die der Sprecher nicht zum Ausdruck bringt, können nicht beschrieben werden. In solchen Fällen ist man bei der Beschreibung auf Hypothesen angewiesen.

Die vier genannten Instanzen, die in der dargestellten Weise aufeinander bezogen sind, lassen sich als das Inventar der Bedeutungskonstante von Kommunikationsverben festhalten. So ergibt sich eine Art von Kernkonzept für die Beschreibung von Kommunikationsverben und von sprachlicher Kommunikation überhaupt. Das bedeutet, daß man die vier Bestandteile der Bedeutungsin- 
variante überall dort auffinden kann, wo mit sprachlichen Mitteln kommuniziert wird. Ganz ähnlich wie hier beschrieben, findet man die Kernbedeutung von verba dicendi auch in anderen Arbeiten, zum Beispiel bei Dirven, Goossens, Putseys und Vorlat (1982), Lehmann (1976), Miller und Johnson-Laird (1976) und Winkler (1987). Ein solches Kernkonzept, das alle Kommunikationsverben erfaßt und das einen für alle gültigen Typ der Bezugnahme auf sprachliche Kommunikationsereignisse darstellt, soll im folgenden allgemeiner Rekurssituationstyp heißen. Hiermit wird ganz global der Typ von Situation beschrieben, in dem Kommunikation eine Rolle spielt. Es handelt sich sozusagen um die allgemeine Bezugssituation von Kommunikations- oder Sprechaktverben, um deren semantische Grundausstattung. Der allgemeine Rekurssituationstyp bildet die Basis für die weitere semantische Differenzierung von Teilmengen von Kommunikationsverben.

\section{Weitere Spezifizierung durch spezielle Rekurssituationstypen}

Aus der Gesamtheit der Kommunikationsverben lassen sich einzelne Gruppen herausgreifen, deren Bedeutungen sich innerhalb eines bestimmten Rahmens bewegen und daher gewisse Ähnlichkeiten aufweisen. Einen solchen Rahmen bilden zusätzliche Parameter, die einzelne Instanzen des allgemeinen Rekurssituationstyps weiter spezifizieren. Diese Parameter können mit unterschiedlichen Werten belegt sein und repräsentieren auf diese Weise einen jeweils anderen Ausschnitt aus der Menge der vorhandenen Verben. (Die Parameter bilden sozusagen die Slots und die einzelnen Werte die Filler, wenn man es in dieser Terminologie ausdrücken will.) Eine mit spezifischen Werten belegte Parametermenge, durch die genau eine bestimmte Menge von Kommunikationsverben erfaßt wird, soll spezieller Rekurssituationstyp heißen.

\subsection{Das Inventar spezieller Rekurssituationstypen}

Welche Elemente des allgemeinen Rekurssituationstyps werden nun solcherart weiter aufgefächert? Für Sprecher und Hörer, das ist leicht einsehbar, besteht diese Notwendigkeit nicht unbedingt; es bleiben also noch die sprachliche Äußerung selbst und die damit verbundene Einstellung des Sprechers. Beide werden mittels verschiedener Parameter einer differenzierteren Betrachtung unterzogen (siehe Abbildung 1).

Die sprachliche Äußerung selbst kann genauer analysiert werden, indem man ihren propositionalen Gehalt näher bestimmt. Ganz global lassen sich zwei Typen von propositionalen Gehalten voneinander differenzieren, die unterschiedlichen Charakter haben, nämlich auf der einen Seite der Mitteilungsgehalt und auf der anderen Seite der Fragegehalt. Der Mitteilungsgehalt stellt sozusagen eine abgeschlossene Proposition dar, die für sich allein stehen kann. 
Der Fragegehalt dagegen bedarf immer einer Ergänzung oder Spezifikation irgendeiner Art. (Dabei ist es gleich, ob es sich um Ergänzungs- oder Entscheidungsfragen handelt.) Er kann in dem Sinne nur schwer allein stehen, sondern verlangt nach einer entsprechenden Antwort, die den Fragegehalt zu einer abgeschlossenen Proposition vervollständigt:

(11) Meine Mutter hat zu mir gesagt: "Paul kommt heute." / daß Paul heute kommt.

(12) Meine Mutter hat mich gefragt: "Wer kommt denn heute?" / wer denn heute kommt.

(13) Meine Mutter hat mich gefragt: "Kommt Paul heute?" / ob Paul heute kommt.

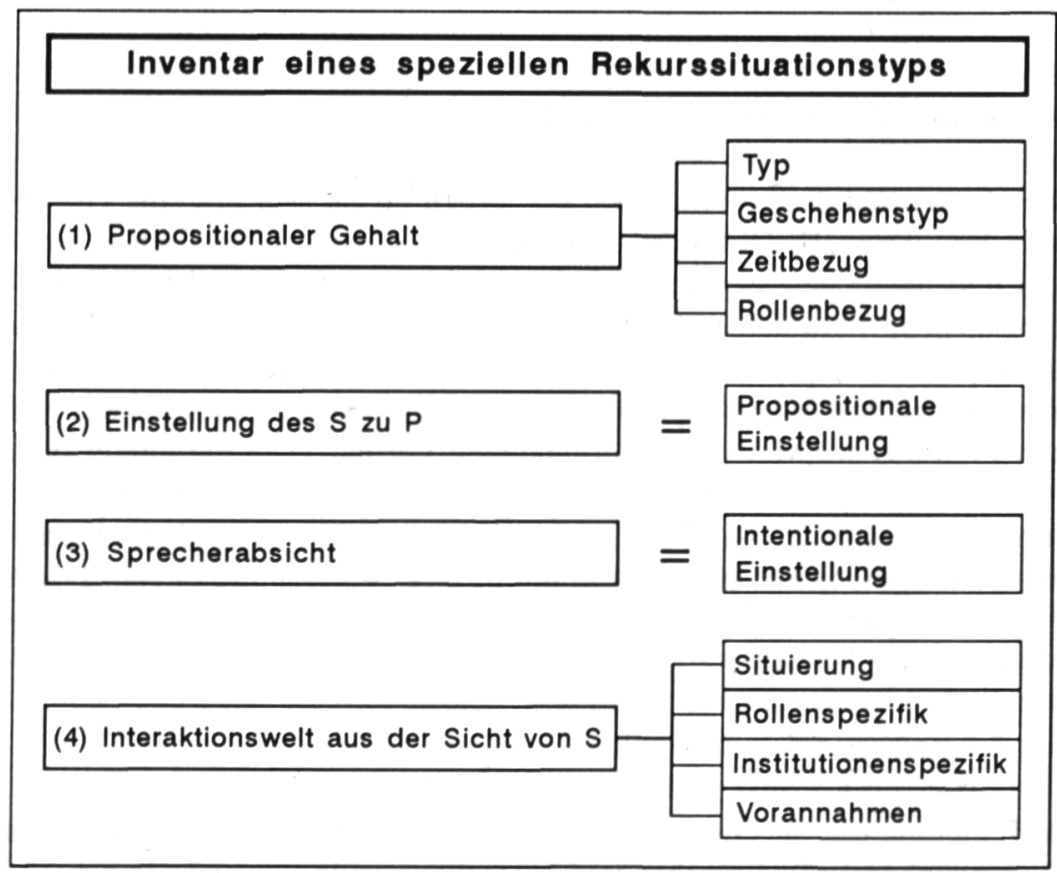

Abbildung 1: Inventar eines speziellen Rekurssituationstyps.

Weiterhin muß man sich klarmachen, welchen Typ von Geschehen der jeweilige propositionale Gehalt repräsentiert, auf welche Rollen (Personen) er sich bezieht und auch, wie der zeitliche Bezug der Äußerung aussieht. Bei den $G e-$ schehenstypen kann man unterscheiden zwischen Ereignis, Zustand, Handlung und unbestimmten Typen, wobei Ereignisse und Zustände mittels der Verben untereinander nur sehr schwer beziehungsweise gar nicht zu differenzieren sind. Eine deutliche Abgrenzung gibt es nur gegenüber den Handlungen. 
(14) Fritz hat erzählt, daß es gestern in Hamburg geregnet hat.

(15) Paul hat sich verpflichtet, für die Bestellung der Hotelzimmer zu sorgen.

Daneben gibt es Verben, bei denen der Geschehenstyp unbestimmt sein kann, das heißt, es sind mehrere Möglichkeiten vorhanden. Beim Zeitbezug des propositionalen Gehaltes existieren alle theoretisch vorhandenen Möglichkeiten, beim Rollenbezug sind ebenfalls die verschiedensten Möglichkeiten denkbar. Soweit zu den Parametern und Werten, die sich auf die sprachliche Äußerung beziehen.

Was die kommunikative Einstellung des Sprechers betrifft, so wird unterschieden zwischen der propositionalen Sprechereinstellung und der intentionalen Einstellung, der Sprecherabsicht. Hinzu kommen weitere Vorannahmen des Sprechers, die die Interaktionswelt aus seiner Sicht konstituieren. Unter den propositionalen Sprechereinstellungen finden sich epistemische, voluntative, evaluative, emotive sowie auch graduierende Einstellungen. Sie sind alle jeweils mit positiven oder negativen beziehungsweise für diesen Bereich relevanten Werten belegt. Bei den emotiven Einstellungen wäre es relativ sinnlos, lediglich eine Positiv-negativ-Differenzierung vorzunehmen; hier werden bestimmte grundlegende Emotionen wie Freude, Ärger, Leid unterschieden. Für die intentionalen Einstellungen stehen Werte zur Verfügung, die die Absichten des Sprechers meist in bezug auf bestimmte Hörerhandlungen beziehungsweise bestimmte Effekte beim Hörer zum Ausdruck bringen. Das heißt, der Hörer soll etwas tun oder unterlassen, er soll etwas zur Kenntnis nehmen, etwas für wahr halten, etwas in bestimmter Weise bewerten und so weiter. Weiterhin trifft der Sprecher eine Reihe von Annahmen über die Kommunikationssituation. Diese Präsuppositionen erfassen ganz unterschiedliche Facetten der Interaktionswelt und sind am variabelsten, da sie unter anderem den gesamten Bereich gesellschaftlicher und individueller Normen und Regeln abdecken müssen. Es können für bestimmte Verben gesonderte Annahmen hinzukommen, die bei anderen überhaupt nicht auftreten müssen. Immer aber wird es wohl Vorannahmen des Sprechers geben, die sich in unterschiedlicher Weise auf den Hörer und sein Verhalten beziehungsweise seine Einstellungen beziehen. Der Sprecher kann zum Beispiel davon ausgehen, daß der Hörer bestimmte Kenntnisse und Fähigkeiten besitzt, daß er Sachverhalte in bestimmter Weise bewertet, und so weiter. Weitere Sprecherpräsuppositionen können die Interessenlage von Sprecher und Hörer bezüglich der Proposition betreffen - bei "versprechen" oder "raten" wird der propositionale Gehalt im Hörerinteresse liegen, bei "bitten" oder "auffordern" eher im Sprecherinteresse - oder sich auch auf den propositionalen Gehalt, den Gang der Dinge selbst beziehen - beispielsweise kann $P$ erwartbar oder nicht erwartbar sein.

Wenn man sich die hier angesetzten Parameter und ihre möglichen Wertebelegungen genauer ansieht, so wird deutlich, daß die dadurch entstehenden Typen von Rekurssituationen in weiten Teilen gängigen Sprechaktklassifizierungen (sowie Klassifikationen von illokutionären Akten) entsprechen, wie sie 
von Austin (1962) und Searle (1969) über Ballmer und Brennenstuhl (1981), Searle und Vanderveken (1985) bis zu Vanderveken (1990, 1991), Ulkan (1992) und anderen immer wieder zu finden sind. Bei den Teilaspekten sprachlichen Wissens, die in den Rekurssituationstypen beschrieben werden, handelt es sich schließlich um das Hintergrundwissen von Sprechakten.

\subsection{Die hierarchische Struktur spezieller Rekurssituationstypen}

Die Bedeutungsgesichtspunkte, die damit in einem speziellen Rekurssituationstyp erfaßt sind, treffen auf jedes einzelne zu diesem Typ gehörende Verb gleichermaßen zu. Spezielle Rekurssituationstypen müssen sich in mindestens einer Parameterbelegung voneinander unterscheiden. Dabei werden Untertypen durch die Belegung mit spezielleren Werten konstituiert; die ihnen übergeordneten Rekurssituationstypen enthalten allgemeinere Parameterbelegungen. So enthält beispielsweise der Rekurssituationstyp Fragen in der Interaktionswelt außer der Situierung "initial" keine weiteren Spezifizierungen. Bei den Prüfungsfragen kommt dann noch eine Rollenspezifik derart hinzu, daß der Sprecher immer der Prüfende und der Hörer in irgendeiner Form der Lernende ist. Ein anderer Fragen-Typ ist stets an mehrere Hörer gerichtet und enthält dieses zusätzliche Merkmal in seiner Rollenspezifik. Reaktive Frageverben sind dagegen in ihrer Situierung eben nicht initial, sondern sie sind Reaktionen auf andere Äußerungen in der jeweiligen Kommunikationssituation. Die genannten Spezifizierungen können sich durchaus auch auf mehrere Werte beziehen. Wie aus dem Gesagten zu ersehen ist, weisen die speziellen Rekurssituationstypen eine hierarchische Ordnung auf; eine in der gleichen Weise hierarchisch geordnete Struktur ist für die ihnen zugehörenden Verbmengen charakteristisch. Für die Direktive ergibt sich beispielsweise das in Abbildung 2 skizzierte Bild.

Die verschiedenen Rekurssituationstypen der Direktive sind so strukturiert, daß sie sich in Fragen, Aufforderungen und Bitten untergliedern, die wiederum weitere Untertypen aufweisen. Die speziellen Rekurssituationstypen charakterisieren zunächst also Typen von Situationen, auf die jeweils mit einer Teilmenge von Kommunikationsverben Bezug genommen wird. Jedem dieser durch eine bestimmte Parametermenge charakterisierten Typen ist genau die Menge von Kommunikationsverben zugeordnet, die der Bezugnahme auf diesen Typ dient. Das heißt, die in den Rekurssituationstypen mit bestimmten Werten belegte Menge von Parametern stellt einen wesentlichen Teil der semantischen Beschreibung der einzelnen Verben dar. Insofern hat die hierarchische Struktur der Rekurssituationstypen ihre Entsprechung in der Struktur der ihnen zugehörigen Menge von Kommunikationsverben.

Der Rekurssituationstyp der Fragen (und mit ihm die entsprechenden Verben) ist unterteilt in Situationen, die charakteristisch sind für Prüfungsfragen, reaktive Fragen und Fragen, die sich an mehrere Hörer wenden. (Entsprechend gibt es Verben, die Bezug auf Prüfungsfragen nehmen, die reaktive Fragen wie- 
dergeben, und so weiter.) $\mathrm{Zu}$ prüfen wäre, ob es sich bei drängendem Fragen oder bei taktisch geschicktem Fragen auch bereits um selbständige Untertypen der Fragen handelt. Bei den Aufforderungen finden sich die Untertypen der Befehle, Aufträge, Weisungen und Verbote mit den jeweils dazugehörigen Verbmengen. Weitere Subtypen lassen sich unter Umständen herausfiltern. Die Bitten sind bislang noch nicht weiter spezifiziert, es lassen sich aber auch hier Untertypen denken, die sich etwa auf die Dringlichkeit der Bitte für den Sprecher und das damit verbundene $\mathrm{Ma} ß$ an Unterwürfigkeit, das er dem Hörer gegenüber zeigt, beziehen. Bei den zugeordneten Verben würde sich das in dem Unterschied zwischen "bitten" und "flehen" niederschlagen.

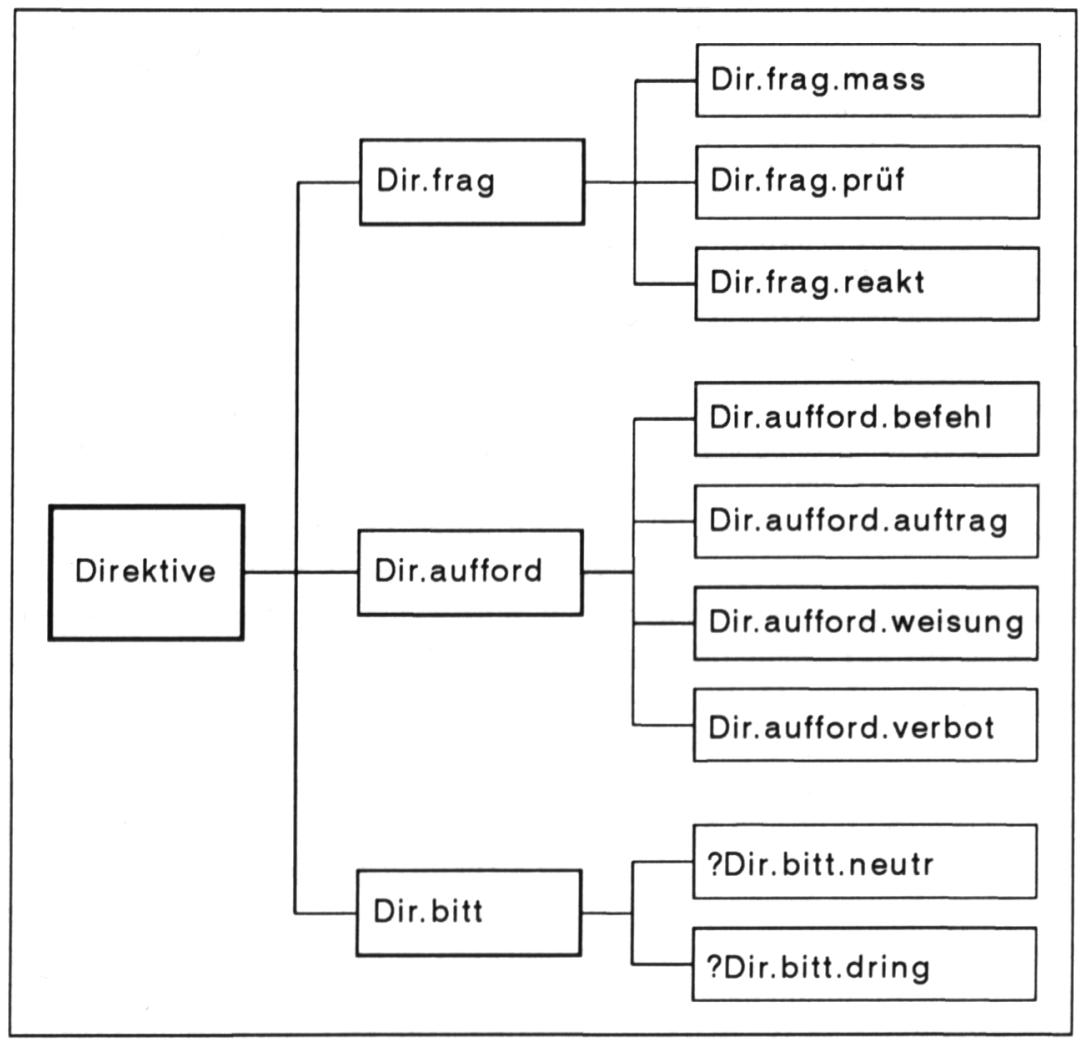

Abbildung 2: Struktur der speziellen Rekurssituationstypen der Direktive.

Die hierarchische Ordnung der speziellen Rekurssituationstypen regelt ihre Beziehungen untereinander. Mit der nächsthöheren Ebene, dem allgemeinen Rekurssituationstyp, sind sie dadurch verbunden, daß ihre Parameter Spezifizierungen einzelner Elemente des allgemeinen Typs sind. Die Beziehung zum einzelnen Kommunikationsverb besteht darin, daß die im Rekurssituationstyp vorhandenen Wertebelegungen quasi das semantische Grundinventar des einzel- 
nen Verbs bilden, das noch durch spezielle Merkmale und Verwendungsbedingungen ergänzt wird.

\section{Wortfelder}

Auf der dritten Beschreibungsebene werden die Beziehungen der Verben eines oder mehrerer verwandter spezieller Rekurssituationstypen zueinander beschrieben. Diese Beziehungen lassen sich in Wortfeldstrukturen darstellen. Wortfelder operieren auf den Werten der speziellen Rekurssituationstypen und ziehen noch weitere Gesichtspunkte hinzu. Die Strukturierung eines Wortfeldes wird durch die jeweils für die beschriebene Verbmenge relevanten Kriterien bestimmt. Diese Kriterien können nun von Wortfeld zu Wortfeld durchaus unterschiedlich sein, generelle Festlegungen lassen sich hier nicht treffen. Ein Beispiel für ein solches Wortfeld, das Lügen-Feld, ist im Beitrag von Storrer (in diesem Band) unter bestimmten Gesichtspunkten bereits näher untersucht worden. Für die Strukturierung dieses Feldes waren die unterschiedliche Thematisierung der einzelnen Argumente seiner Verben und der Grad der Intensität der Lüge ausschlaggebend. Das letzte Kriterium regelt die Beziehung zwischen "lügen", "schwindeln" und "flunkern", das erstgenannte unterscheidet die unpräfigierten und die mit verschiedenen Präfixen versehenen Verbformen voneinander. Dabei ist noch anzumerken, daß die Präfigierungen mit "rum-" und "zusammen-" und die reflexiven, mit "durch-" präfigierten Verben sich auf den Verlauf der Handlung beziehen, während durch die übrigen Präfixformen jeweils andere Argumente des Verbs in den Vordergrund gerückt werden.

Kandidaten für die Kriterien, die ein Wortfeld strukturieren, sind zum Beispiel die syntaktisch-semantischen Belegungsmöglichkeiten der Verben (Realisierung von Sprecher, Hörer und Proposition), bestimmte ihrer Bedeutungsmerkmale oder auch Intensitäts- oder graduelle Unterschiede im Hinblick auf einen einzelnen Gesichtspunkt. Welche dieser Kriterien relevant werden, muß für jedes einzelne Wortfeld neu überdacht werden. Hierzu ist anzumerken, daß kleinere Wortfelder oft keine sehr starke Strukturierung aufweisen werden.

Die Vorgehensweise ist im weiteren so, daß man entweder die Verbmenge eines einzelnen speziellen Rekurssituationstyps in einem Wortfeld erfaßt oder aber auch die Kommunikationsverben mehrerer hierarchisch geordneter Rekurssituationstypen zu Wortfeldern zusammenfaßt, also beispielsweise alle Fragen-Verben, alle Auffordern-Verben oder möglicherweise sogar alle Direktive. Die Anordnung der einzelnen Verben im Wortfeld müßte dann den hierarchischen Strukturen der Rekurssituationstypen entsprechen. Bei Wortfeldern eines solchen Umfangs wie dem aller Direktive besteht indes leicht die Gefahr, daß sie unübersichtlich werden. Außerdem kann die Zahl der zu berücksichtigenden strukturierenden Kriterien zu umfangreich ausfallen, was ebenfalls die Darstellung sehr stark beeinträchtigt beziehungsweise sogar unmöglich macht. 
Ein Wortfeld kann nun als eine Art von Baum- oder auch Netzstruktur dargestellt werden. Der Platz des einzelnen Kommunikationsverbs innerhalb dieses Baumes oder Netzes wird bestimmt durch eine Menge von Kriterien beziehungsweise von Werten, die ein einzelnes Kriterium, beispielsweise der Intensitätsgrad, annehmen kann. Diese Menge von Werten und Kriterien kann in Form eines Merkmalbündels angegeben werden. Zu jedem Verb gehört ein solches Merkmalbündel, das sich in mindestens einem Punkt von dem jedes Verbs eines anderen Knotens unterscheiden muß.

Gewisse Ähnlichkeiten mit dieser Wortfeldstruktur weist das im Beitrag von Fellbaum (in diesem Band) vorgestellte semantische Netz WordNet auf. Auch dort werden die Wörter beziehungsweise Begriffe durch lexikalische und semantische Relationen miteinander verbunden. Die Menge von Wörtern, die einen bestimmten Begriff lexikalisiert, wird zu einem sogenannten Synset zusammengefaßt, was in bestimmtem Maße mit den speziellen Rekurssituationstypen vergleichbar ist. Relationen wie der Hyponymie, Synonymie oder Antonymie wird auch in WordNet Rechnung getragen.

\section{Die Beschreibung der einzelnen lexikalischen Einheiten in Lexikoneinträgen}

Die niedrigste und nur ein Verb umfassende Ebene innerhalb dieser Darstellung ist der Lexikoneintrag für jedes Kommunikationsverb mit seinen individuellen Besonderheiten und Lesarten, wobei natürlich anzumerken ist, daß die verschiedenen Lesarten eines Verbs oft unterschiedlichen Rekurssituationstypen angehören und somit auch unterschiedliche Lexikoneinträge erhalten. Ein derartiger Lexikoneintrag umfaßt mehr als die bloße Beschreibung der Verbbedeutung. Diese ist nur ein Punkt unter anderen. In sehr starkem Maße werden dort die Gebrauchsbedingungen der Verben berücksichtigt, die in mehreren Bestandteilen des Lexikoneintrags ihren Niederschlag finden. Der Lexikoneintrag eines Verbs enthält folgende Positionen:

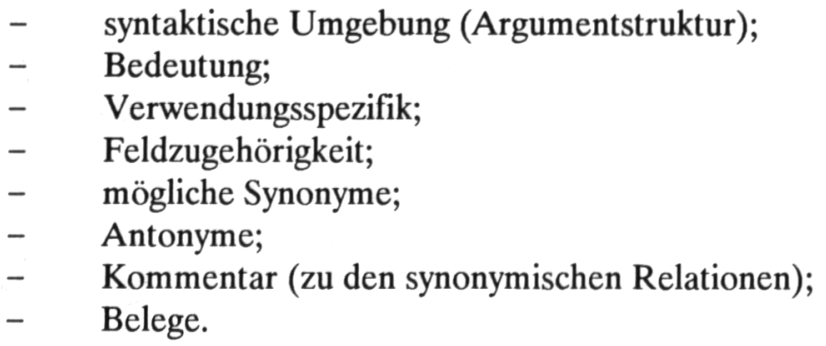

Unterziehen wir nun diese einzelnen Bestandteile des Lexikoneintrags einer näheren Betrachtung. Er enthält als erstes Angaben zur syntaktischen Umgebung eines Verbs. Hier werden die syntaktischen Argumentstellen des Verbs einmal 
bezüglich ihrer Obligatheit beziehungsweise Fakultativität und zum anderen bezüglich ihrer möglichen Realisierungsformen festgehalten. Das geschieht in Form einer Paraphrase beziehungsweise eines vereinfachten Subkategorisierungsmerkmals. Zusätzliche Merkmale, die nicht den Status von Argumenten haben, wie die verschiedenen Formen der Passivfähigkeit des Verbs, die Unmöglichkeit oder Notwendigkeit der direkten Rede oder auch Angaben über eine besondere Reihenfolge im Satz werden gesondert festgehalten.

Ein zweiter Bestandteil des Lexikoneintrags ist die eigentliche Bedeutungsbeschreibung des Verbs. Sie besteht zum einen aus einer lexikographischen $\mathrm{Pa}$ raphrase, die kurz und prägnant die Bedeutung umschreibt, und zum anderen aus einer ausführlichen Beschreibung der Verbbedeutung. Diese erfolgt unter explizitem Bezug auf die in den speziellen Rekurssituationstypen vorkommenden Parameter und Werte. Über jene Bedeutungsbestandteile hinaus, die durch den jeweiligen Rekurssituationstyp bestimmt sind, werden an dieser Stelle aber auch die ganz spezifischen Besonderheiten des einzelnen Verbs beziehungsweise einer seiner Lesarten notiert. Dadurch erhält man die Möglichkeit, ein einzelnes Verb von allen anderen Verben desselben Rekurssituationstyps abzuheben und in seiner Spezifik darzustellen.

Der weiteren Differenzierung der einzelnen Verben dient auch eine detaillierte Darstellung der Verwendungsbedingungen des Verbs, die darüber hinaus noch Angaben zu syntaktisch-semantischen Besonderheiten bei der Thematisierung und Realisierung der Argumente, Angaben zu Modifikatoren und Kollokationen sowie Angaben zur stilistischen Einordnung und zu regionalen Besonderheiten sowie zur explizit performativen Verwendbarkeit des Verbs enthält. Wichtigster Teil einer solchen Operation bleibt aber die genaue Beschreibung der Gebrauchsbedingungen, da aus der vorgenommenen Beschreibung dann auch Generalisierungen gewonnen werden können, die unter Umständen nicht nur für das einzelne Verb von Belang sind. (Keller [in diesem Band] sieht die Gebrauchsregeln für ein sprachliches Zeichen, die im Regelfall konventionalisiert sind, als dessen Bedeutung an.) Für die Darstellung der Gebrauchsbedingungen werden zunächst möglichst detailliert die Situationen beschrieben, in denen das betreffende Verb verwendet werden kann. Weiter wird festgehalten, welche Aspekte dieser Situation durch das Verb besonders hervorgehoben werden, ob es Besonderheiten oder Einschränkungen bei der Verwendung seiner einzelnen Argumente gibt, ob die Verwendung des Verbs auf bestimmte Bereiche beschränkt bleibt und vieles andere mehr. Stellt man einen Vergleich dieser Beschreibungen von allen Verben eines Typs an, so kann man feststellen, ob es so etwas wie generelle Gebrauchsbedingungen beziehungsweise generelle Einschränkungen der Gebrauchsbedingungen für die zu einem speziellen Rekurssituationstyp gehörende Verbmenge gibt.

Weitere Bestandteile eines Lexikoneintrags sind die Angaben von Synonymen und Antonymen des betreffenden Verbs. Antonyme werden hier entsprechend gängigen Klassifizierungen eingeteilt; siehe zum Beispiel Agricola und Agricola (1992), verschiedene Arbeiten von Cruse (1976, 1980, 1986), Geckeler 
(1980), Herberg (1992), Lang (1995) und Müller (1989). Synonymie wird als eine skalare Eigenschaft der Verwendung von Verben begriffen, die mehr oder weniger stark ausgeprägt sein kann. (Zu dieser Synonymieauffassung erscheint ein Forschungsbericht von Harras [i. Vorb.]; zu anderen Synonymieauffassungen vergleiche unter anderem Bickmann [1978], Görner \& Kempcke [1973], Herberg [1992] und Wolski [1989]). Synonymie ist also graduell zu sehen. Schwache Synonymie beschränkt sich dementsprechend im wesentlichen auf die Gemeinsamkeit der typkonstituierenden Bedeutungsmerkmale von Verben. Das heißt zum Beispiel, daß alle Verben, die zu den verschiedenen Untertypen der Aufforderungen gehören, untereinander schwach synonym sind, und zwar unter dem alle diese Typen konstituierenden Bedeutungsgesichtspunkt, daß ein Sprecher sich mit einer sprachlichen Äußerung an einen Hörer wendet, um zu bewirken, daß der Hörer eine bestimmte, in der Äußerung benannte Handlung ausführt oder gegebenenfalls auch unterläßt. Der Sprecher geht selbstverständlich von der Annahme aus, daß der Hörer dazu auch in der Lage ist. Entsprechendes gilt für Fragen und alle anderen Typen. Bei starken Synonymiebeziehungen müssen noch weitere, spezifischere Bedeutungsgemeinsamkeiten hinzukommen. Den verschiedenen Arten der Synonymiebeziehungen ist im Lexikoneintrag ein spezieller Kommentar gewidmet, in dem anhand von Ersetzungsbeispielen gezeigt wird, wie die Verwendungskontexte für starke oder schwache Synonyme aussehen müssen. Ausnahmen und Abweichungen werden an dieser Stelle erläutert und begründet.

Ein Lexikoneintrag enthält also eine große Menge recht unterschiedlicher Informationen, von denen aber der überwiegende Teil einen Bezug zu den $\mathrm{Pa}-$ rametern der speziellen Rekurssituationstypen und letztendlich auch zum allgemeinen Rekurssituationstyp hat. Jedes Verb stellt eine spezielle Ausprägung der Elemente dieser Typen dar.

Damit ist die eine Richtung der Bedeutungsbeziehungen benannt. Die Bestandteile des allgemeinen und speziellen Rekurssituationstyps gehen praktisch in jedes Kommunikationsverb ein. Aber es läßt sich auch die umgekehrte Richtung angeben; nicht von ungefähr ist den Gebrauchsbedingungen der Verben in den Lexikoneinträgen so breiter Raum gewährt worden. Zum einen soll ja beispielsweise auch die Synonymierelation als eine Eigenschaft der Verwendung von Verben begriffen werden, und zum anderen kann man aus einer Menge von Gebrauchsbedingungen für die verschiedenen Verben eines Rekurssituationstyps Generalisierungen ableiten, die wiederum auf die Strukturierung der Rekurssituationstypen zurückwirken. Hierfür ein Beispiel aus dem Bereich der Fragen-Verben: Unterzieht man die Verwendungsspezifik solcher Verben wie "aushorchen", "ausloten", "ausforschen", "auskundschaften", "abklopfen", "erforschen", "entlocken", "herauslocken" und "eruieren" einer genaueren Betrachtung, so wird man feststellen, daß alle diese Verben nicht nur auf eine einfache Fragehandlung Bezug nehmen, sondern immer auch ein taktisches Vorgehen des Sprechers einbeziehen. Dieser zusätzliche Gesichtspunkt könnte gegebenenfalls zur Konstituierung eines weiteren Untertyps von Fragen führen. Vor- 
stellbar ist weiterhin, daß es zu Generalisierungen über mehrere Typen kommen kann. Auch die Phänomene, die Storrer (in diesem Band) behandelt, können zu Generalisierungen zum Beispiel über bestimmte Verbpräfigierungen führen, und zwar letztendlich auf der Grundlage einer genauen Beschreibung der Gebrauchsregeln der einzelnen Verben.

\section{Zum Verhältnis von Sprechakten und Sprechaktverben}

Ein Punkt, der in engem Zusammenhang mit der Beschreibung von Kommunikations- oder Sprechaktverben - letztere bilden eine Teilklasse der erstgenannten - steht und der einem dabei immer wieder ins Blickfeld gerät, ist das Verhältnis zwischen kommunikativen Handlungen und Kommunikationsverben, zwischen Sprechakten und Sprechaktverben. Mit anderen Worten, das Problem der Lexikalisierungsmöglichkeiten kommunikativer Handlungen spielt bei der Beschreibung dieses Faktenbereichs eine große Rolle. Um die Quintessenz, die sich jeder leicht denken kann, gleich vorwegzunehmen: Es besteht keine 1:1Entsprechung zwischen Sprechakten und Sprechaktverben. Man kann aber wohl davon ausgehen, daß sich das Ungleichgewicht mehr zu einer Seite hin verlagert, und zwar insofern, als es nicht für jeden Sprechakt eine Lexikalisierung im verbalen Bereich gibt. Der umgekehrte Fall einer vorhandenen Lexikalisierung ohne zugeordneten Sprechakt ist, falls er überhaupt auftritt, viel seltener. Jedenfalls sind die Fälle hier nicht so klar wie in der anderen Richtung.

Man kann nun folgende Fragen anschließen: Für welche Sprechakte gibt es Lexikalisierungen in Form von Sprechaktverben und für welche nicht? Sind es bestimmte Ebenen oder Bereiche, für die solche Lexikalisierungen existieren? Wenn ja, um welche handelt es sich dabei und warum werden gerade diese lexikalisiert? Und umgekehrt, gibt es Fälle, für die ein Kommunikationsverb existiert, das aber keine Entsprechung in einem Sprechakt hat?

Beginnen wir mit dem letzteren. Ein solcher Fall ist schwer vorstellbar. Denn mit einem Kommunikationsverb nimmt man ja immer Bezug auf eine Äußerungssituation und auf eine sprachliche Äußerung, so daß eigentlich immer auch ein Sprechakt vorliegen müßte. Es kann hier also nur um Grenz- oder mehrdeutige Fälle gehen. So existiert eine ganze Anzahl von Verben, die nur in einer ihrer Lesarten als Kommunikationsverb Verwendung finden können. Bei ihnen kann es zu solchen Grenzfällen kommen. Andere Verben nehmen hingegen Bezug auf komplexere Akte, die entweder mehrere Sprechakte umfassen oder aber noch über den eigentlichen Sprechakt hinausgehende Bestandteile enthalten.

Beispielsweise ist also die Grenzziehung zwischen Kommunikations- und Kognitionsverben nicht immer einfach. Es gibt eine ganze Reihe von Verben, die eigentlich einen kognitiven Akt, einen mentalen Vorgang bezeichnen, aber auch als Kommunikationsverb gebraucht werden, wobei sie dann zum Ausdruck bringen, daß jemand das Ergebnis dieses kognitiven Akts äußert. 
(16) Der Referent schlußfolgerte aus dieser Tatsache, daß man für eine dauerhafte Lösung des Problems eng mit anderen Industriezweigen zusammenarbeiten müsse.

Wenn also jemand schlußfolgert, vollzieht er streng genommen nicht den Sprechakt des Schlußfolgerns, sondern den des Mitteilens des Ergebnisses seiner Schlußfolgerungen. Der kognitive Akt ist aber sozusagen als dessen Voraussetzung immer in dem Sprechakt enthalten. Das Sprechaktverb "schlußfolgern" (Schlußfolgerungen äußern) präsupponiert das kognitive Verb "schlußfolgern" (Schlußfolgerungen ziehen). Solche und ähnliche Verben bewegen sich in den Grenzgebieten zu benachbarten Verbklassen.

Anders gelagert sind nun jene Fälle, in denen das Verb mehr als nur einen Sprechakt bezeichnet. Ich meine hier Verben wie "diskutieren", "sich unterhalten" und ähnliche. Es gibt keinen einzelnen Sprechakt des Diskutierens oder des Sich-Unterhaltens. Es handelt sich immer um größere Sequenzen, die aus mehreren, ganz unterschiedlichen Sprechakten bestehen können.

(17) Sie diskutierten lange über die Interpretation ihrer Versuchsergebnisse.

(18) Karl unterhält sich mit Anna über seine letzte Urlaubsreise nach Australien.

In einer Unterhaltung können Fragen, Mitteilungen und auch Aufforderungen vorkommen. Dabei werden Informationen ausgetauscht, Meinungen geäußert, Bewertungen vorgenommen, Geschichten erzählt und vieles andere mehr. All das ergibt keinen einheitlich strukturierten Sprechakt, der den Typ "Sich unterhalten" repräsentieren würde, ganz abgesehen von der Tatsache, daß sich hier mindestens zwei Sprecher den Äußerungspart teilen müssen. Ganz ähnlich verhält es sich beim Diskutieren: Hier werden Standpunkte dargelegt, Argumente vorgebracht und widerlegt, Gegenargumente aufgestellt, Beweise angeführt, Problemlösungen gesucht und anderes mehr. In beiden Fällen entstehen im Ergebnis sehr komplexe Sprechakte, die sich aus vielen einzelnen Teilsprechakten zusammensetzen, auf deren Gesamtheit aber mit einem einzigen Kommunikationsverb Bezug genommen wird. Bei Bedarf kann man sich natürlich auch auf die einzelnen Teilakte beziehen, dann aber jeweils mit den spezifischen Verben.

Damit vergleichbar sind in gewisser Weise Verben, die noch anderes als nur einen Sprechakt bezeichnen, das heißt Verben, die sich nicht ausschließlich auf Sprachliches beziehen. Zu denken ist hier an Verben wie "aushorchen" oder "entlocken".

(19) Andersdenkende wurden immer wieder ausgehorcht.

(20) Sie entlockte ihm schließlich doch das streng gehütete Familiengeheimnis. 
Solche Verben nehmen nicht einfach nur Bezug auf den Sprechakt des Fragens und spezifizieren auch nicht nur eine bestimmte Art des Fragens wie etwa "nachfragen", "interviewen", "konsultieren", "anfragen" oder "sich durchfragen". Die gesamte Handlung, auf die sie sich beziehen, läßt sich zwar als Fragehandlung klassifizieren, umfaßt aber mehr als eine bloße Frage, von der Tatsache, $\mathrm{da} ß$ auch hier mehrere sprachliche Äußerungen erfolgen müssen, einmal abgesehen. Diese Verben bezeichnen nicht nur den oder die Äußerungsakt(e), sie bezeichnen gleichzeitig auch die Vorgehensweise des Sprechers. Um die gewünschten Auskünfte zu erhalten, muß der Sprecher taktisch klug vorgehen. Das heißt, er wird vielleicht oftmals gar nicht fragen, sondern nur den Gesprächsverlauf und die Wahl der Themen so geschickt steuern, daß er allein schon auf diese Weise zu den von ihm gewünschten Informationen kommt. Oder aber er fragt nur sehr indirekt beziehungsweise nach vermeintlich ganz anderen Dingen. So werden "aushorchen" oder "entlocken" zu sehr komplexen Handlungen, die in diesem Sinne keine Entsprechung in einem singulären Sprechakt haben.

\subsection{Möglichkeiten der Lexikalisierung von kommunikativen Handlungen durch Verben}

Wenden wir uns hier zunächst den Fällen zu, in denen es Sprechakte gibt, für die keine Lexikalisierungen in Form von Sprechaktverben existieren. Wie auch im Vorangegangenen strebe ich keine Vollständigkeit der Darstellung an, sondern greife nur einzelne Beispiele heraus. Sehen wir uns zunächst zwei Fälle etwas genauer an. Da wäre als erstes die Lexikalisierung von Negationen. Hier sind maximal drei Stufen lexikalisiert. Zuerst besteht die positive Ausgangsstufe (äußern, daß p), die mit einem Verb wie "behaupten" lexikalisiert wird. Auf einer zweiten Stufe kann die Negation stehen (äußern, daß $\neg$ p), was einem Verb wie "widersprechen" gleichkommt. Eine dritte Stufe wäre nun "äußern, daß $\neg(\neg p)$ ", das heißt soviel wie "doch p" und wird ausgedrückt durch ein Verb wie "beharren auf". Theoretisch sind ja nun noch weitere Stufen denkbar. Es könnte also die nunmehr positive Proposition wiederum negiert werden, und so weiter. Dafür gibt es dann allerdings keine weiteren Lexikalisierungen mehr. Das bedeutet, es kann nur bis zu einem bestimmten Komplexitätsgrad lexikalisiert werden. Alles, was über die dritte Stufe hinausgeht, ist zu umfangreich, um mit einem einzelnen Verb beschrieben zu werden. Dieser komplexe Sachverhalt muß dann auch sprachlich komplexer, das heißt mit mehreren Wörtern oder in einer längeren Umschreibung, ausgedrückt werden. Hinzu kommt wahrscheinlich noch, daß die Fälle bis zur zweiten Negation wesentlich häufiger vorkommen als alles, was darüber hinausgeht. So ist es sicher ein generelles, rein sprachökonomischen Gründen geschuldetes Prinzip, daß selten vorkommende Sachverhalte und Entitäten eher sprachlich umschrieben werden, als daß für sie 
eine eigene Lexikalisierung zur Verfügung gestellt wird. Häufig Vorkommendes wird dagegen eher lexikalisiert.

Sehen wir uns als zweites Beispiel die Frageverben und die Frageakte etwas genauer an. Hier läßt sich eindeutig feststellen, daß es viel mehr Arten von Frageakten gibt als Frageverben; es bleibt herauszufinden, welche Bereiche lexikalisiert sind und welche nicht.

So gibt es beispielsweise kein Verb für rhetorisches Fragen. Diese Besonderheit muß im Satz stets zusätzlich geäußert werden beziehungsweise mit einer gesonderten Äußerung angeschlossen werden. Für deliberative Fragen gibt es die Lexikalisierung "sich fragen". Dagegen haben andere Arten von Fragen wie zum Beispiel Regiefragen, parlamentarische Anfragen, bestimmte Fragen im pädagogischen Bereich, Erinnerungsfragen und viele andere mehr keine spezifischen Lexikalisierungen im verbalen Bereich. Will man auf sie Bezug nehmen, so benutzt man einfach das neutrale und allgemeine (und damit auch sehr breit anwendbare) "fragen". Es gibt sogar mehrere Lexikalisierungen, die mehr oder weniger nur eine ganz allgemeine, relativ undifferenzierte Fragebedeutung repräsentieren, nämlich "fragen", "sich erkundigen", "sich informieren". Mit diesen drei Verben kann man sich auf alle Arten von Fragen beziehen, eben weil sie keine so spezifizierte Bedeutung haben.

Welche zusätzlichen Aspekte können nun darüber hinaus lexikalisiert werden? Es gibt spezielle Verben für reaktives Fragen - normalerweise ist Fragen ja ein initialer Akt - wie zum Beispiel "rückfragen", "nachfragen", "zurückfragen". Auch für Prüfungsfragen gibt es eigene Lexikalisierungen; diese sind "abfragen", "examinieren", "prüfen" und "abhören". Ebenso existieren spezifische Verben für den Fall, daß eine oder mehrere Fragen nicht nur an einen einzelnen Hörer, sondern an eine Menge von Hörern gerichtet sind. Hierher gehören Verben wie "herumfragen", "rumfragen", "rundfragen" und "sich durchfragen". Eine andere Gruppe von Verben, die man auch unter Fragen subsumieren kann, ist bereits genannt worden. Es handelt sich um jene Verben, die ein taktisch geschicktes Fragen benennen, um so zu Informationen zu kommen, die man auf eine einfache und direkte Nachfrage hin nicht bekommen hätte. Das sind Verben wie "aushorchen", "ausforschen", "auskundschaften", "ausbaldowern", "ausloten", "entlocken", "herauslocken" und eventuell noch einige andere wie "erkunden", "ausspionieren", "erforschen" und "eruieren".

Wenn das Fragen besonders drängend, sich ständig wiederholend und mitunter sogar belästigend für den Hörer ist, gibt es dafür auch gesonderte Verben, zum Beispiel "bedrängen", "ausfragen", "ausquetschen", "bestürmen", "bohren", "drängen", "drängeln", "löchern", "auf jemanden einstürmen". Soll durch die Frage(n) eine Sache vollständig und gründlich geklärt werden, kann man das ebenfalls mit eigenen Verben ausdrücken, etwa mit "klären", "aufklären", "ergründen" oder "abklären".

Bestimmte institutionalisierte Verwendungen von Fragen sind natürlich auch lexikalisiert, und zwar durch Verben wie "verhören", "vernehmen", "interviewen", "konsultieren". Allerdings gibt es bei diesen Lexikalisierungen keine 
weiteren Spezifikationen. $\mathrm{Ob}$ es sich um polizeiliche, richterliche oder andere Verhöre beziehungsweise Vernehmungen handelt, muß separat ausgedrückt werden. Auch ob sich das Konsultieren auf den juristischen, den medizinischen oder den universitären Bereich ("einen Rechtsanwalt/einen Arzt/einen Professor konsultieren") erstreckt, ist nicht lexikalisiert. Ähnlich allgemein ist "interviewen" festgelegt. Mit diesem Verb wird lediglich ausgedrückt, daß es sich in irgendeiner Form um institutionalisiertes Fragen handelt. Es kann jedoch nicht lexikalisiert werden, welche Medien hier welche Art von Interview führen.

Abbildung 3 vermittelt eine Übersicht darüber, für welche Teilbereiche aus der Gesamtmenge der Fragen Lexikalisierungen im verbalen Bereich existieren.

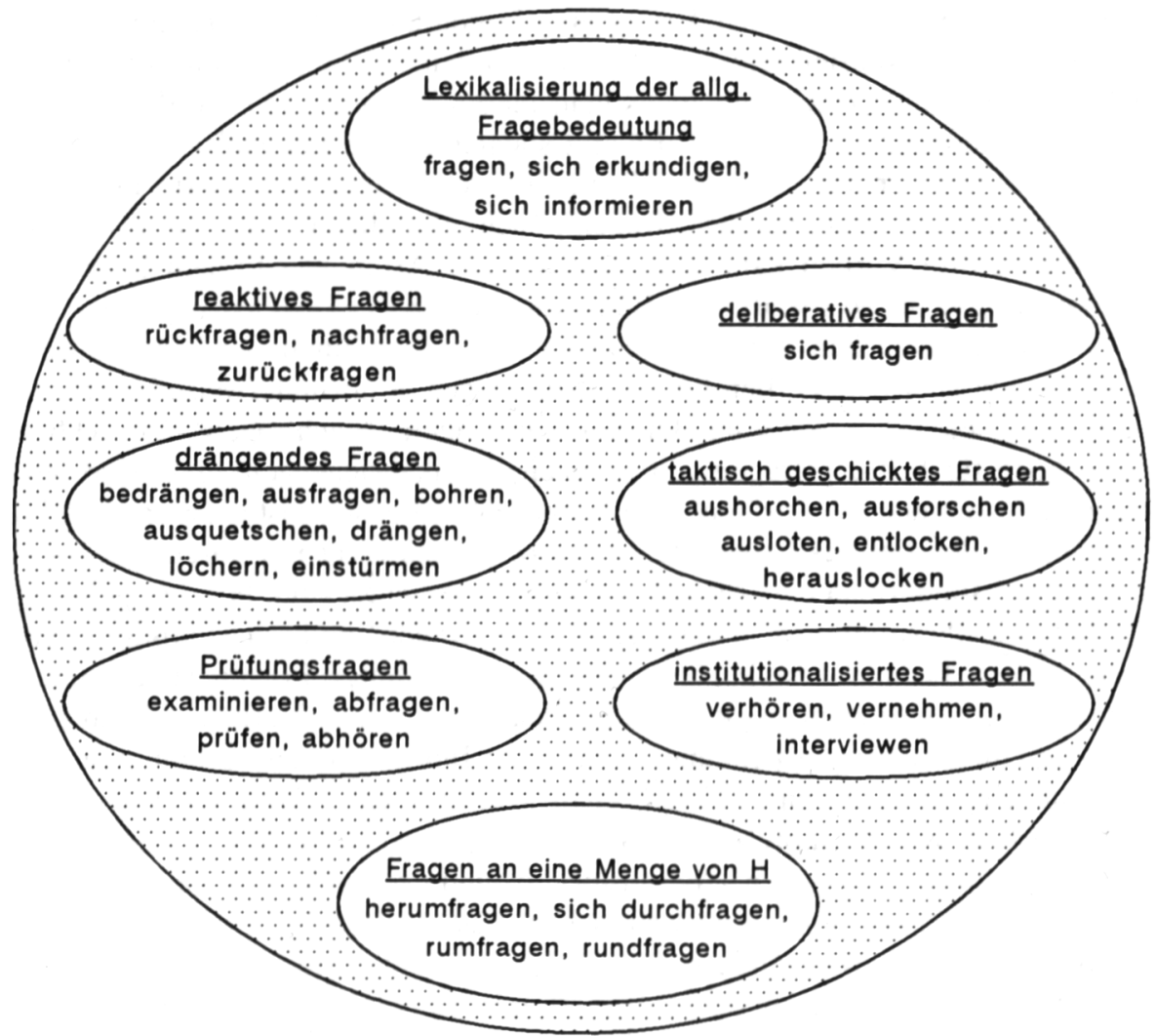

Abbildung 3: Möglichkeiten der Lexikalisierung von Fragen durch Verben.

Es besteht kaum die Möglichkeit, das Medium oder den Kanal des Fragens im Verb selbst zu lexikalisieren. Ob es sich um eine telefonisch, brieflich, persönlich, mündlich, schriftlich oder sonstwie gestellte Frage handelt, muß mit anderen sprachlichen Mitteln als dem Verb ausgedrückt werden. 
Der Kanal wird ohnehin nur dann lexikalisiert, wenn dieser Aspekt einer Äußerung im Vordergrund steht, das heißt, wenn er für den Sprecher der Äußerung, in der das Sprechaktverb verwendet wird, besonders wichtig ist und hervorgehoben werden soll. Natürlich kann es auch durch die Situation bedingt sein, wie etwas geäußert wird, wie zum Beispiel in (21). Die Art der Äußerung tritt dann in den Hintergrund, beziehungsweise es ist möglich, jede beliebige Äußerung mit diesem Verb zu beschreiben. Es können also sowohl Aussagen oder Fragen als auch Aufforderungen oder anderes geflüstert, gebrüllt oder auch beim Telefonieren mitgeteilt werden:

(21) "Wie heißt denn der Schauspieler, der gerade auf die Bühne kommt?", flüsterte er ihr ins Ohr.

(22) Sie flüsterte ihm mühsam beherrscht zu, er solle sofort den Raum verlassen.

(23) Er brüllte, daß er sich über so einen Vorfall gar nicht aufregen könne.

Prinzipiell wird immer derjenige Gesichtspunkt lexikalisiert, auf dem das größte Gewicht liegt. Natürlich geschieht das immer aus der Sicht desjenigen, der auf die Äußerung und die Äußerungssituation Bezug nimmt. Eine gleichzeitige Lexikalisierung mehrerer unterschiedlicher Aspekte scheint indes kaum möglich zu sein. So existiert beispielsweise kein Verb, das ein flüsterndes, drängendes Fragen bezeichnet oder ein briefliches Beschreiben eines Sachverhaltes. Auch telefonische Lügen, mündliche Versprechen oder lautstarkes Klagen sind nicht lexikalisiert.

Weitere Beispiele für die Möglichkeiten der Lexikalisierung lassen sich in anderen Gruppen von Sprechaktverben finden. Beispiele für Lexikalisierungslücken in unterschiedlichen Bereichen und mögliche Ansätze zu ihrer Erklärung finden sich bei Harras (1994). Im großen und ganzen kann man wohl sagen, daß die Lexikalisierungen zunächst auf einem bestimmten Basisniveau angelegt sind, von dem aus dann gegebenenfalls noch Spezifizierungen vorgenommen werden. Spezielle Aspekte werden jedoch auch nur insoweit lexikalisiert, als sie noch verallgemeinerbar sind. Je spezifischer die auszudrückenden Bedingungen sind, desto seltener erfolgt die Lexikalisierung durch ein gesondertes Verb. In solchen Fällen werden dann andere, in der Regel komplexere sprachliche Mittel eingesetzt.

\section{Fazit}

Für alle vorhandenen Kommunikations- oder Sprechaktverben läßt sich so etwas wie eine Kernbedeutung isolieren. Die einzelnen Verben können auf der Grundlage dieser Kernbedeutung auf verschiedenen Ebenen und unter verschiedenen Gesichtspunkten detaillierter beschrieben werden. Zwischen den einzelnen Ebenen besteht ein Zusammenhang derart, daß die Bedeutungsge- 
sichtspunkte der allgemeineren Ebenen immer auch Bestandteil der spezifischeren Ebenen sind, und umgekehrt lassen sich aus den Bedeutungsbeschreibungen der einzelnen Verben teilweise auch allgemeinere Parameter generalisieren. Hierbei spielen die Gebrauchsbedingungen der Verben eine zentrale Rolle. Aus einer Menge von Gebrauchsbedingungen für (bis zu einem gewissen Grade ähnliche) einzelne Verben können Generalisierungen - wenn man so will: konventionalisierte Gebrauchsbedingungen - abgeleitet werden. Keller (1995, S. 77) findet für diesen und ähnliche Zusammenhänge eine sehr treffende und griffige Formulierung, wenn er sagt, da $\beta$ Begriffe »die geistigen Korrelate unserer Gebrauchsregeln « sind und »im allgemeinen von diesen erzeugt « werden. Begriffe sind gewissermaßen eine Abstraktion aus einer Summe von Gebrauchsregeln und -bedingungen für sprachliche Zeichen. Solche Abstraktionen und Generalisierungen schlagen sich im Rahmen des hier vorgestellten Beschreibungsmodells auf der Ebene der speziellen Rekurssituationstypen nieder. Die größtmögliche Abstraktion für den dargestellten Lexikonausschnitt stellt der allgemeine Rekurssituationstyp dar. Diese beiden Ebenen sind begriffliche Ebenen von unterschiedlichem Allgemeinheitsgrad. Der allgemeine Rekurssituationstyp liefert praktisch einen generellen Begriff von Kommunikationsverben. Die speziellen Rekurssituationstypen entsprechen spezifischeren und somit eingeschränkteren Begriffen von Untermengen von Kommunikationsverben. Auf der Ebene der Lexikoneinträge wird ausschließlich die Bedeutung einzelner lexikalischer Einheiten beschrieben. Die Beziehung zu den eher begrifflichen Ebenen wird zwar stets hergestellt, was aber explizit beschrieben wird, ist die Verbbedeutung. Auf der Grundlage dieser Bedeutung sind die Verben in der Lage, Begriffe auszudrücken.

Die unterschiedlichen Verben lexikalisieren jeweils ein semantisches Grundinventar aus den Rekurssituationstypen sowie darüber hinausgehende spezifische Bedeutungsgesichtspunkte. Dabei wurde festgestellt, daß die Lexikalisierung spezifischer Aspekte immer nur bis zu einem Niveau erfolgt, das noch einen bestimmten Grad an Verallgemeinerung erlaubt. Ganz individuelle Besonderheiten werden nicht mehr lexikalisiert. Es gibt so etwas wie eine grundlegende Ebene von Lexikalisierung, auf der das Basiswissen und die Basisstrukturen von Kommunikation versprachlicht sind, und von dort ausgehend können noch einige andere wichtige Bedeutungsgesichtspunkte lexikalisiert werden. Diese weiterführenden Verbalisierungen sind zum Teil quantitativ (in der Vorkommenshäufigkeit bestimmter Bedeutungsaspekte), zum Teil qualitativ (in der Wichtigkeit bestimmter Bedeutungsaspekte für den Kommunikationszusammenhang) und zum Teil sicher auch sprachökonomisch begründet. 


\section{Literatur}

Agricola, E. \& Agricola, C. (1992). Wörter und Gegenwörter (Duden-Taschenbücher, Band 23). Mannheim: DUDEN-Verlag.

Austin, J. L. (1962). How to do things with words. Oxford: Oxford University Press.

Ballmer, Th. \& Brennenstuhl, W. (1981). Speech act classification (Springer Series in Language and Communication 8). Heidelberg: Springer.

Bickmann, H.-J. (1978). Synonymie und Sprachverwendung. Verfahren zur Ermittlung von Synonymenklassen als kontextbeschränkten Äquivalenzklassen (Reihe Germanistische Linguistik 11). Tübingen: Niemeyer.

Cruse, D. A. (1976). Three classes of antonym in English. Lingua, 38, 281-292.

Cruse, D. A. (1980). Antonyms and gradable complementaries. In D. Kastovsky (Hrsg.), Perspektiven der lexikalischen Semantik (Gesamthochschule Wuppertal, Schriftenreihe Linguistik, Band 2) (S. 14-25). Bonn: Bouvier.

Cruse, D. A. (1986). Lexical semantics. Cambridge: Cambridge University Press

Dirven, R., Goossens, L., Putseys, Y. \& Vorlat, E. (1982). The scene of linguistic action and its perspectivization by speak, talk, say and tell (Pragmatics \& Beyond III: 6). Amsterdam: Benjamins.

Fellbaum, Ch. (in diesem Band). WordNet: Ein semantisches Netz als Bedeutungstheorie (S. 211-230).

Geckeler, H. (1980). Die Antonymie im Lexikon. In D. Kastovsky (Hrsg.), Perspektiven der lexikalischen Semantik (Gesamthochschule Wuppertal, Schriftenreihe Linguistik, Band 2) (S. 42-69). Bonn: Bouvier.

Görner, H. \& Kempcke, G. (Hrsg.). (1973). Synonymwörterbuch. Sinnverwandte Ausdrücke der deutschen Sprache. Leipzig: VEB Bibliographisches Institut.

Harras, G. (1991). Konzeption zu einer erklärenden Synonymik kommunikativer Ausdrücke des Deutschen. Unveröffentlichtes Manuskript. Mannheim: Institut für deutsche Sprache.

Harras, G. (1994). Situationstypen und lexikalische Konzepte (SFB 245, Finanzierungsantrag 1995 - 1997). Unveröffentlichtes Manuskript. Mannheim: Institut für deutsche Sprache.

Harras, G. (i. Vorb.). Synonymik und Synonymie. Tübingen: Narr.

Harras, G. \& Winkler, E. (1994). A model for describing speech act verbs. The semantic base of a polyfunctional dictionary. In W. Martin, W. Meijs, M. Moerland, E. ten Pas, P. van Sterkenburg \& P. Vossen (Hrsg.), Euralex 1994. Proceedings (S. 440-448). Amsterdam.

Herberg, D. (1992). Makrostrukturelle Beziehungen im Wortschatz und in Wörterbucheinträgen. Möglichkeiten und Grenzen des allgemeinen einsprachigen Wörterbuchs. In U. Brauße \& D. Viehweger (Hrsg.), Lexikontheorie und Wörterbuch (Lexicographica, Series Major, Band 44) (S. 90-163). Tübingen: Niemeyer.

Keller, R. (1995). Zeichentheorie. Zu einer Theorie semiotischen Wissens. Tübingen: Francke.

Keller, R. (in diesem Band). Begriff und Bedeutung (S. 47-66).

Lang, E. (1995). Das Spektrum der Antonymie. Semantische und konzeptuelle Strukturen im Lexikon und ihre Darstellung im Wörterbuch. In G. Harras (Hrsg.), Die Ordnung der Wörter (Institut für deutsche Sprache, Jahrbuch 1993) (S. 30-98). Berlin: de Gruyter.

Lehmann, D. (1976). Untersuchungen zur Bezeichnung der Sprechaktreferenz im Englischen (Forum Linguisticum Band 8). Bern: Lang.

Miller, G. A. \& Johnson-Laird, P. N. (1976). Language and perception. Cambridge, MA: The Belknap Press of Harvard University Press.

Müller, W. (1989). Die Antonymie im allgemeinen einsprachigen Wörterbuch. In F. J. Hausmann et al. (Hrsg.), Wörterbücher - Dictionaries - Dictionnaires. Ein internationales Handbuch zur Lexikographie (HSK 5.1) (S. 628-635). Berlin: de Gruyter.

Searle, J. R. (1969). Speech acts. Cambridge: Cambridge University Press. 
Searle, J. R. \& Vanderveken, D. (1985). Foundations of illocutionary logic. Cambridge: Cambridge University Press.

Storrer, A. (in diesem Band). Verbbedeutung und Situationsperspektivierung (S. 231 - 255).

Ulkan, M. (1992). Zur Klassifikation von Sprechakten (Linguistische Arbeiten 174). Tübingen: Niemeyer.

Vanderveken, D. (1990/1991). Meaning and speech acts (2 vols). Vol. 1: Principles of language use. Vol. 2: Formal semantics of success and satisfaction. Cambridge: Cambridge University Press.

Winkler, E. (1987). Syntaktische und semantische Eigenschaften von verba dicendi. Unveröffentlichte Dissertation, Berlin.

Winkler, E. (1995). Die Darstellbarkeit lexikalischen Wissens - am Beispiel kommunikativer Ausdrücke des Deutschen. In G. Harras (Hrsg.), Die Ordnung der Wörter (Institut für deutsche Sprache, Jahrbuch 1993) (S. 328-354). Berlin: de Gruyter.

Wolski, W. (1989). Die Synonymie im allgemeinen einsprachigen Wörterbuch. In F. J. Hausmann et al. (Hrsg.), Wörterbücher - Dictionaries - Dictionnaires. Ein internationales Handbuch zur Lexikographie (HSK 5.1) (S. 614-628). Berlin: de Gruyter. 